DESY 01-161

HU-EP-01/42

October 2001

\title{
Continuum limit in abelian projected SU(2) lattice gauge theory *
}

\author{
V. Bornyakov ${ }^{\mathrm{a}}{ }^{\dagger}$ and M. Müller-Preussker ${ }^{\mathrm{b}}$ \\ a NIC/DESY Zeuthen, Platanenallee 6, 15738 Zeuthen, Germany \\ b Humboldt-Universität zu Berlin, Institut für Physik, 10115 Berlin, Germany
}

We study the continuum limit of the abelian string tension and the density of abelian monopoles calculated after carefully fixing the maximal abelian gauge by employing the simulated annealing algorithm. We present the evidence that the abelian string tension converges to the nonabelian one in the continuum limit. For the monopole density we confirm earlier findings that the density of the properly defined infrared monopoles has correct scaling while the total density seems divergent in the continuum limit due to ultraviolate contributions. We also compare with results obtained with the usual iterative gauge fixing algorithm.

\section{INTRODUCTION}

Numerous lattice studies have provided support for the dual superconductor scenario of confinement 11. Most of the results have been obtained in the maximal abelian gauge (MAG) at particular values of the lattice spacing. To our knowledge no attempt was made for extrapolation into the continuum limit. In this paper we want to fill in this gap.

\section{GAUGE FIXING AND SIMULATION DETAILS}

We work with $S U(2)$ gluodynamics in the MAG which is defined by maximizing the functional [2]

$F\left(U^{g}\right)=\frac{1}{8 L^{4}} \sum_{x, \mu} \operatorname{Tr}\left[U_{x, \mu}^{g} \sigma_{3} U_{x, \mu}^{g, \dagger} \sigma_{3}\right]$

with respect to the gauge transformations $g_{x}$ : $U_{x, \mu}^{g}=g_{x} U_{x, \mu} g_{x+\mu}^{\dagger}$. The abelian projection $U_{x, \mu} \rightarrow u_{x, \mu}$ is defined by the following relations

$U_{x, \mu}^{i i}=\cos \left(\phi_{x, \mu}\right) e^{i \theta_{x, \mu}^{i}}, \quad \theta_{x, \mu}^{1}=-\theta_{x, \mu}^{2} \equiv \theta_{x, \mu}$

$u_{x, \mu} \equiv \operatorname{diag}\left\{e^{i \theta_{x, \mu}}, e^{-i \theta_{x, \mu}}\right\}$.

It is well known that the functional (11) has many local maxima corresponding to Gribov copies [3].

*Poster presented by V. Bornyakov at Latice 2001, Berlin.

${ }^{\dagger}$ On leave of absence from IHEP, Protvino, Russia
Table 1

\begin{tabular}{|c|c|c|c|}
\hline & $\beta=2.4$ & $\beta=2.5$ & $\beta=2.6$ \\
\hline$L=L_{s, t}$ & 24 & 24 & 28 \\
$a$ & $0.12 \mathrm{fm}$ & $0.085 \mathrm{fm}$ & $0.06 \mathrm{fm}$ \\
$L \cdot a$ & $2.9 \mathrm{fm}$ & $2.1 \mathrm{fm}$ & $1.7 \mathrm{fm}$ \\
$\left\langle F>_{S A}\right.$ & $.7336(1)$ & $.7511(1)$ & $.7662(1)$ \\
$\left\langle F>_{R O}\right.$ & $.7310(1)$ & $.7491(1)$ & $.7648(1)$ \\
\hline
\end{tabular}

The standard procedure consists in selecting a local maximum one finds more or less randomly by an iterative algorithm. Our prescription of gauge fixing is to find the global maximum of (1D). This prescription resembles the minimal Landau gauge [4]. The 'global maxima' definition of gauge fixing is known to be a limit of the global gauge fixing introduced in 河. As a numerical tool we use the simulated annealing (SA) algorithm [6.7]. This algorithm helps to get closer to the global maxima but at some low auxiliary temperature $T$ it, nevertheless, gets trapped into metastable states of the corresponding spin glass. To overcome this problem we applied the procedure to every MC configuration 10 times by starting from different gauge copies and used the copy with maximal value of $F(U)$. For comparison we carried out gauge fixing with the standard iterative algorithm with alternating relaxation and over-relaxation sweeps (RO algorithm). Only one gauge copy was considered as it is commonly done. 
The simulation details are presented in Table 1. There we also show the average value of $F(U)$ for the two algorithms. We investigated 20 statistically independent configurations per $\beta$-value.

\section{MONOPOLE DENSITY}

We use the DeGrand-Toussaint definition of magnetic currents [8]

$k_{x, \mu}=\frac{1}{4 \pi} \varepsilon_{\mu \nu \rho \sigma} \partial_{\nu} \bar{\theta}_{x, \rho \sigma}$

with $\quad \bar{\theta}_{x, \nu \mu}=\partial_{\mu} \theta_{x, \nu}-\partial_{\nu} \theta_{x, \mu}+2 \pi m_{x, \mu \nu} \quad$ and $-\pi \leq \bar{\theta}_{n, \mu \nu}<\pi, \quad m_{n, \mu \nu}=0, \pm 1, \pm 2$.

The physical monopole density is then defined as

$\rho_{\text {mon }}=\frac{1}{4\left(a L_{s}\right)^{3} L_{t}}\left\langle\sum_{x, \mu} k_{x, \mu}\right\rangle$.

Following [9] we will call monopoles relevant for confinement 'infrared' (IR) monopoles and the rest of monopoles, normally forming small loops, 'ultraviolate' (UV) ones. The corresponding densities are denoted as $\rho_{\operatorname{mon}}^{I R, U V}$. In 10] it has been found that monopoles from the largest cluster (LC) are sufficient to reproduce the string tension. This observation suggests that the IR monopoles can be identified as the monopoles belonging to the LC [9]. However, as it has been indicated in 10] the LC may split into parts if the volume is not large enough. We confirm this observation. Moreover, we have found that, when the LC splits, then there usually appear clusters with nontrivial winding

$w_{\mu}=\frac{1}{L_{\mu}} \sum_{k_{x, \mu} \in \text { cluster }} k_{x, \mu}$.

Therefore, we suggest the following definition of IR monopoles: monopoles from LC plus those from clusters with nontrivial $w_{\mu}$. In Fig. 1 we present our results for $\rho_{\text {mon }}^{I R, U V}$, measured according to the new definition. [ We also show our results for the density of monopoles belonging to the LC, $\rho_{\text {mon }}^{L C}$, together with data from [10]. We

3 Throughout this work we use the following values of the nonabelian string tension $\sqrt{\sigma a^{2}}$-11]: $0.266(2)$ at $\beta=2.4$; $0.1905(8)$ at $\beta=2.5 ; 0.136(4)$ at $\beta=2.6$. We set the scale putting $\sqrt{\sigma}=440 \mathrm{Mev}$.

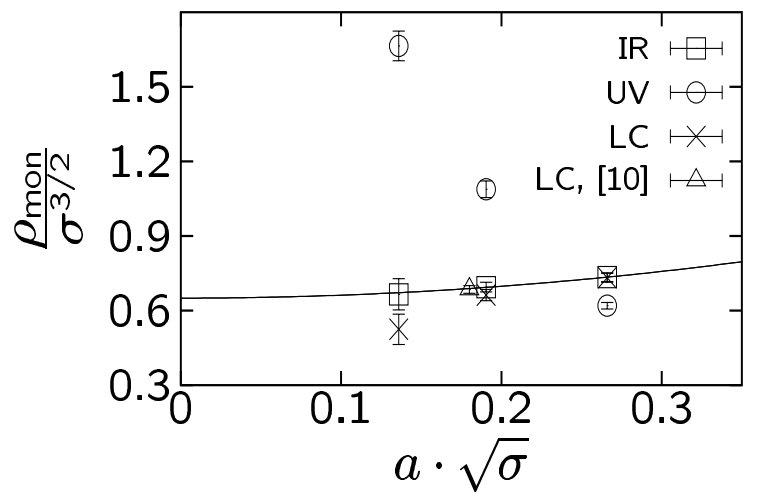

Figure 1. Density of monopoles vs. lattice spacing.

found that when the lattice size is large enough the clusters with nontrivial windings are rare and the two definitions give coinciding results. This is also supported by the agreement between our $\rho_{\text {mon }}^{I R}$ value obtained at $\beta=2.5, L \cdot a=2.1 \mathrm{fm}$ and the $\rho_{\text {mon }}^{L C}$ value from [10] obtained at $\beta=$ $2.5115, L \cdot a=2.8 \mathrm{fm}$. We conclude that the new definition of IR monopoles can be successfully used on lattices with size $L \cdot a \geq 1.7 \mathrm{fm}$. It is to be checked, how this definition works on smaller lattices. We extrapolated $\rho_{\text {mon }}^{I R}$ to the continuum limit using a quadratic fit (solid curve in Fig. 1). We find that $\rho_{\text {mon }}^{I R}=0.65(2) \sigma^{3 / 2}$. Data in Fig. 1 show that while $\rho_{m o n}^{I R}$ is finite, $\rho_{m o n}^{U V}$ seems to diverge providing a divergent total density. Our data indicate that $\rho_{\text {mon }}^{U V} / \sigma^{3 / 2} \sim 1 / \sqrt{\sigma a^{2}}$.

\section{ABELIAN STATIC POTENTIAL AND STRING TENSION}

We introduce the abelian Wilson loop

$W_{a b}(C)=\frac{1}{2} \operatorname{Tr}\left(\prod_{l \in C} u_{l}\right)$

and the abelian static potential

$V_{a b}^{T}(R)=\log \left(\frac{\left\langle W_{a b}(R, T)\right\rangle}{\left\langle W_{a b}(R, T+1)\right\rangle}\right)$.

On-axes as well as $(0,1,1)$ off-axes directions were used. We apply smearing for spatial abelian links to improve the signal-to-noise ratio. $V_{a b}^{T}(R)$ has been fitted to a constant within the range 


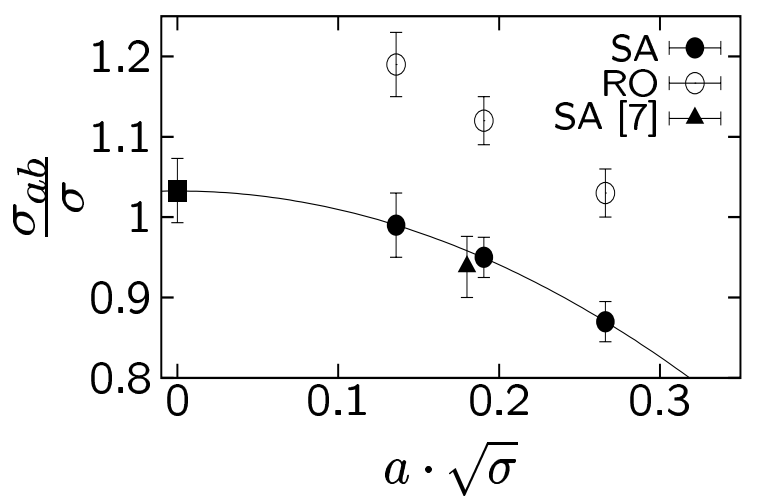

Figure 2. Ratio of the abelian and nonabelian string tensions vs. lattice spacing for $S A$ and $R O$ algorithms. The solid line represents a quadratic fit. For comparison data from [7] are shown.

$5 \leq T a \leq 8$ to obtain $V_{a b}(R)$. We employ a dimensionless form of the usual parametrization for the static potential

$\frac{V_{a b}(R)}{\sqrt{\sigma}}=\frac{V_{0}}{\sqrt{\sigma}}-\frac{e}{R \sqrt{\sigma}}+\frac{\sigma_{a b}}{\sigma} R \sqrt{\sigma}$.

Data points were fitted to this form in the range $0.5<R \cdot \sqrt{\sigma}<2.5$. Fig. 2 presents our main result. The continuum limit of $\sigma_{a b}$ agrees with that of $\sigma$ within error bars. One can see a good agreement with a result from [7] obtained by the same method. It is worth noting that our result is still biased by Gribov copies effects, although these effects were substantially reduced in comparison with the common procedure. Using results of [7] we can estimate the bias as $\Delta \sigma_{a b} / \sigma \sim-0.03$. Taking into account this bias would not spoil our conclusion.

We also made an attempt to compute the charge-two abelian static potential. In [7]:12 it has been conjectured that the charge-two abelian string tension $\sigma_{a b}^{q=2}$ is equal to the adjoint string tension. If this is true, then in the continuum limit $\sigma_{a b}^{q=2} / \sigma \rightarrow 8 / 3$. Unfortunately, our statistics appeared to be insufficient to provide a reliable estimation of $\sigma_{a b}^{q=2}$. The work on this and also on the monopole static potential is in progress.

\section{CONCLUSIONS}

- With Gribov copies effects taken under better control we found that in the continuum limit $\sigma_{a b} / \sigma=1.03(4)$ supporting abelian dominance for the string tension within the maximal abelian gauge.

- The density of IR monopoles turns out finite in the continuum limit: $\rho_{\text {mon }}^{I R}=$ $0.65(2) \sigma^{3 / 2}$. Thus, the average distance between monopoles can be estimated as $d_{m o n}=1.15(1) / \sqrt{\sigma}=0.52(1) \mathrm{fm}$.

This work is partially supported by INTAS grant 00-00111. V.B. acknowledges support from RFBR grants 99-01230a and 01-02-17456.

\section{REFERENCES}

1. G. 't Hooft, in 'High Energy Physics', Proceedings of the EPS Int. Conf., Palermo 1975, ed. A. Zichichi; S. Mandelstam, Phys. Rep. C23 (1976) 245.

2. A.S. Kronfeld, M.L. Laursen, G. Schierholz and U.-J. Wiese, Phys. Lett. 198B (1987) 516.

3. V.N. Gribov, Nucl. Phys. B139 (1978) 1.

4. D. Zwanziger, Nucl. Phys. B412 (1994) 657.

5. C. Parrinello and G. Jona-Lasinio, Phys. Lett. B251 (1990) 175; D. Zwanziger, Nucl. Phys. B345 (1990) 461.

6. S. Kirkpatrick, C.D. Gelatt Jr. and M.P. Vecchi, Science 220 (1983) 671; V. Cerny, J. Opt. The. Appl. 45 (1985) 41.

7. G.S. Bali, V. Bornyakov, M. Müller-Preussker and K. Schilling, Phys. Rev. D54 (1996) 2863.

8. T.A. DeGrand and D. Toussaint, Phys. Rev. D22 (1980) 2478.

9. V. Bornyakov, M. Chernodub, F. Gubarev, M. Polikarpov, T. Suzuki, A. Veselov and V. Zakharov, hep-lat/0103032.

10. A. Hart and M. Teper, Phys. Rev. D58 (1998) 014504; Phys. Rev. D60 (1999) 114506.

11. J. Fingberg, U.M. Heller and F. Karsch, Nucl. Phys. B392 (1993) 493.

12. G. Poulis, Phys. Rev. D54 (1996) 6974. 